\title{
A single-arm Phase II validation study of preventing oxaliplatin-induced hypersensitivity reactions by dexamethasone: the AVOID trial
}

This article was published in the following Dove Press journal:

Drug Design, Development and Therapy

12 November 2015

Number of times this article has been viewed

\author{
Yoichiro Yoshida' \\ Keiji Hirata ${ }^{2}$ \\ Hiroshi Matsuoka ${ }^{3}$ \\ Shigeyoshi Iwamoto ${ }^{4}$ \\ Masahito Kotaka ${ }^{5}$ \\ Hideto Fujita ${ }^{6}$ \\ Naoya Aisu' \\ Seiichiro Hoshino' \\ Takeo Kosaka ${ }^{6}$ \\ Kotaro Maeda ${ }^{3}$ \\ Fumiaki Kiyomi $^{7}$ \\ Yuichi Yamashita' \\ 'Department of Gastroenterological \\ Surgery, Fukuoka University \\ Faculty of Medicine, Fukuoka, Japan; \\ ${ }^{2}$ Department of Surgery, Fukuoka \\ Sanno Hospital, Fukuoka, Japan; \\ ${ }^{3}$ Department of Surgery, Fujita \\ Health University School of Medicine, \\ Toyoake, Japan; ${ }^{4}$ Department of \\ Surgery, Kansai Medical University \\ Hirakata Hospital, Osaka, Japan; \\ ${ }^{5}$ Gastrointestinal Cancer Center, Sano \\ Hospital, Kobe, Japan; ${ }^{6}$ Department \\ of Surgical Oncology, Kanazawa \\ Medical University, Uchinada, Japan; \\ ${ }^{7}$ Academia, Industry and Government \\ Collaborative Research Institute \\ of Translational Medicine for Life \\ Innovation, Fukuoka University, \\ Fukuoka, Japan
}

Background: Patients with colorectal cancer treated with oxaliplatin are at risk of hypersensitivity reactions, with the incidence estimated to be $12 \%-20 \%$. Coinfusion of dexamethasone and oxaliplatin could potentially reduce the incidence of these reactions, but oxaliplatin is reported to be incompatible with alkaline compounds in solution. However, in a previous retrospective study we found that the $\mathrm{pH}$ of a solution of dexamethasone and oxaliplatin was less than 7.4, and that hypersensitivity to oxaliplatin could have been prevented by coinfusion of dexamethasone. We aimed to evaluate the effectiveness of coinfusion of dexamethasone and oxaliplatin to prevent oxaliplatin-induced hypersensitivity reactions.

Patients and methods: The AVOID trial was a prospective, multicenter, open-label, singlearm Phase II trial conducted from January to September 2013. The study included 73 patients who received capecitabine plus oxaliplatin (XELOX) or XELOX plus bevacizumab therapy for colorectal cancer. In all patients, oxaliplatin was administered in combination with dexamethasone. The primary outcome measure was the presence of hypersensitivity reactions.

Results: Hypersensitivity reactions occurred in three patients (4.1\%); all three experienced a cutaneous reaction (grade 1 erythema). None of the 73 patients developed respiratory symptoms, ocular symptoms, or anaphylaxis. Grade 3 or higher hemotoxicity occurred in $13.7 \%$ of the patients and grade 3 or higher nonhematological toxicity occurred in $13.7 \%$. The response rate to treatment was $64.4 \%$.

Conclusion: The coinfusion of dexamethasone and oxaliplatin effectively reduced oxaliplatininduced hypersensitivity reactions in patients with colorectal cancer. This approach should be considered for all patients treated with oxaliplatin, allowing treatment to be completed as planned.

Keywords: oxaliplatin, dexamethasone, hypersensitivity, allergy

\section{Introduction}

Oxaliplatin, a platinum-based chemotherapeutic agent, is effective for the treatment of advanced colorectal cancer. ${ }^{1-5}$ Unlike other platinum derivatives, oxaliplatin does not cause significant renal impairment or ototoxicity. The dose-limiting toxicity of oxaliplatin is related to peripheral nerve function and the potential development of peripheral neuropathy. ${ }^{6}$

Chemotherapy for metastatic colorectal cancer has remarkably advanced with the introduction of folinic acid, fluorouracil, and irinotecan (FOLFIRI) and folinic acid, fluorouracil, and oxaliplatin (FOLFOX) regimens. However, both these regimens require the implantation of a central venous $(\mathrm{CV})$ access port and treatment is occasionally interrupted by complications associated with the $\mathrm{CV}$ port, such as infection, thrombosis, and pinch-off syndrome. ${ }^{7}$ With the recent development of capecitabine plus oxaliplatin
Correspondence: Yoichiro Yoshida Department of Gastroenterological Surgery, Fukuoka University Faculty of Medicine, 7-45-I Nanakuma, Jonan-ku, Fukuoka 8|4-0|80, Japan

Tel +81928011011

Fax +8I 928639759

Email yy4160@yahoo.co.jp 
(XELOX) therapy, the implantation of a CV port can now be avoided. However, phlebitis caused by peripheral intravenous infusion of antineoplastic agents can limit the completion or continuation of chemotherapy. Factors that contribute to the development of phlebitis include the $\mathrm{pH}$ and osmotic pressure of the solution, the size of the vein used, the size and material of the catheter, and infusion periods. ${ }^{8}$ A number of methods for reducing the risk of phlebitis have been reported, but none are completely effective. ${ }^{9,10}$ In one study, the addition of steroids to the oxaliplatin drip infusion was useful in controlling vascular pain. ${ }^{11}$ However, the addition of steroids can increase the $\mathrm{pH}$ of the solution and affect the stability of oxaliplatin. Furthermore, the prescribing information states that oxaliplatin is incompatible in solution with alkaline medications or media; therefore, it is not mixed with these solutions or simultaneously administered through the same infusion line. Because intravenous dexamethasone (DEX) is alkaline, it is routinely administered to patients before chemotherapy.

To date, there are limited reports on the effectiveness of oxaliplatin combined with steroids. However, we have previously reported that the $\mathrm{pH}$ of a solution of DEX and oxaliplatin was less than 7.4. In that retrospective study, we found that hypersensitivity reactions (HSRs) to oxaliplatin could have been prevented by the coinfusion of DEX. ${ }^{12}$ Little information is available about the risk factors of oxaliplatin-induced HSR. ${ }^{13}$ To our knowledge, only four investigations have previously attempted to identify potential predictive factors by multivariate analysis. ${ }^{14-17} \mathrm{Kim}$ et al reported the possible association between oxaliplatin-induced HSR and clinical characteristics and found that females, younger patients, and patients receiving oxaliplatin in salvage regimens were more likely to experience oxaliplatin-induced HSR. ${ }^{16,17}$ On the basis of multivariate analysis, Mori et al ${ }^{15}$ analyzed that being young and having an oxaliplatin-free interval were independent risk factors, whereas Shao et $\mathrm{al}^{14}$ found higher oxaliplatin dose per infusion as an independent risk factor. Moreover, a recent study reported that a lower dose of DEX $(<20 \mathrm{mg})$ could be a positive risk factor. ${ }^{16}$

To our knowledge, there have been no reports about prospective trials of preventing oxaliplatin-induced HSRs by the coinfusion of DEX. The present prospective study was undertaken to evaluate the effectiveness of DEX to prevent HSRs caused by the administration of oxaliplatin.

\section{Patients and methods}

\section{Study design}

The AVOID trial was a prospective, multicenter, open-label, single-arm Phase II trial in Japan. This study was performed in accordance with the ethical guidelines for clinical studies. The institutional review board at Fukuoka University approved the protocol and the study is registered with the University Hospital Medical Information Network Clinical Trials Registry (ID: UMIN000009742).

This study evaluated the effectiveness of coinfusion of DEX and oxaliplatin to prevent oxaliplatin-induced HSRs. The primary endpoints were to document the incidence of oxaliplatin HSRs of all grades. Secondary endpoints were objective tumor response rate and safety. The target sample size was 70 patients, assuming that the expected HSRs and threshold HSRs were $4 \%$ and $12 \%$, respectively, with a onesided alpha-level of $5 \%$ and a power of $80 \%$.

\section{Patients and eligibility criteria}

Seventy-three patients were enrolled in this study between January and September 2013. Patients were recruited from Fukuoka University Hospital, Fukuoka Sanno Hospital, Fujita Health University Hospital, Kansai Medical University Hirakata Hospital, Sano Hospital, and Kanazawa Medical University Hospital. Eligible patients were $\geq 20$ years of age, with histologically confirmed colorectal cancer without prior chemotherapy for metastatic disease, and with at least 6 months elapsed from completion of any adjuvant therapy. They also met the following criteria: Eastern Cooperative Oncology Group performance status 0-2, life expectancy $\geq 12$ weeks, neutrophil count $\geq 1,000 / \mathrm{mm}^{3}$, platelet count $\geq 75,000 / \mathrm{mm}^{3}$, hemoglobin $\geq 8.0 \mathrm{~g} / \mathrm{dL}$, total bilirubin $\leq 2.0 \mathrm{mg} / \mathrm{dL}$, and serum creatinine $\leq 1.5$ times the upper limit of normal. The institutional review boards of all participating institutions approved the clinical study. Written informed consent was obtained from each patient.

Patients with any of the following conditions were excluded: severe peripheral sensory neuropathy (PSN), a history of serious hypersensitivity to drugs, active infection, paralytic or mechanical bowel obstruction, uncontrolled hypertension, uncontrolled diabetes, cirrhosis, clinically significant cardiovascular disease, history of myocardial infarction within the previous 3 months, uncontrolled angina pectoris or arrhythmia, multiple primary cancers within the past 5 years, pleural effusion requiring drainage ascites or pericardial effusion, uncontrolled diarrhea, clinically significant mental or psychological disease, and any other condition making a patient unsuitable for this study.

\section{Treatment}

The 73 study patients received either XELOX or XELOX plus bevacizumab therapy (oxaliplatin $130 \mathrm{mg} / \mathrm{m}^{2} \pm$ bevacizumab 
$7.5 \mathrm{mg} / \mathrm{kg}$ on day 1 , plus capecitabine $1,000 \mathrm{mg} / \mathrm{m}^{2}$ twice daily on days $1-14$, every 3 weeks) for advanced or recurrent colorectal cancer. All patients received intravenous DEX $(6.6 \mathrm{mg})$ and a selective 5-hydroxytryptamine-3 receptor antagonist before oxaliplatin infusion. In addition, DEX $(6.6 \mathrm{mg})$ was administered in combination with oxaliplatin (Figure 1). ${ }^{18-20}$ Dose reductions were required for all grade 3 or 4 toxicities attributed to the study medications. The dose of bevacizumab was not reduced. Treatment was continued until disease progression, unacceptable toxicities, or withdrawal of consent.

Study treatment was delayed if any of the following criteria were applicable on the day when administration was scheduled or the previous day: a neutrophil count of $<1,000 / \mathrm{mm}^{3}$, a platelet count of $<75,000 / \mathrm{mm}^{3}$, active infection with fever $\geq 38.0^{\circ} \mathrm{C}$, grade 2 or worse diarrhea, grade 3 or worse PSN, and other grade 2 or worse nonhematological toxicities. The oxaliplatin dose was reduced to $100 \mathrm{mg} / \mathrm{m}^{2}$ if grade 3-4 neutropenia or thrombocytopenia, persistent grade 2 or reversible grade 3 PSN, or any grade 3-4 nonhematological toxicities occurred. The study was terminated if grade 3 toxicity persisted after a 21-day washout period or if grade 4 PSN or a grade 2-4 allergic reaction occurred. The study was also terminated if the patient required longer than 3 weeks to recover from an adverse event.

\section{Evaluation of chemotherapy}

All patients underwent physical examination, chest radiography, and computed tomographic scans of the abdomen, pelvis, and chest before starting the treatment. All patients were included in safety and efficacy analyses. The severity of adverse events was evaluated according to the National Cancer Institute Common Toxicity Criteria (NCI-CTC), version 4.0. Tumors were measured at 6- to 8-week intervals, and response was evaluated according to the response evaluation criteria for solid tumors (RECIST) version 1.1. The evaluation of response was based on radiologist-reported measurements. Complete (CR) and partial response (PR) required subsequent confirmation after an interval of at least 4 weeks. The disease control rate (DCR) was calculated from the number of patients who achieved a CR, PR, or stable disease (SD) with treatment, while the overall response rate was based on the number of patients who had CR or PR.

\section{Definition of HSRs}

Drug HSRs are the adverse effects of pharmaceutical formulations (including active drugs and excipients) that clinically resemble allergy. ${ }^{21}$ For general communication, when an allergic drug reaction is suspected, HSR is the preferred term, because true drug allergy and nonallergic HSR may be difficult to differentiate based on the clinical presentation alone, especially in cases of acute severe HSR. ${ }^{22}$ In this study, oxaliplatin-induced hypersensitivity was defined as the occurrence of cutaneous symptoms (such as urticaria, rash, and angioedema), respiratory symptoms (such as dyspnea, bronchospasm, and laryngeal edema), gastrointestinal symptoms, neurological symptoms, or anaphylaxis subsequent to oxaliplatin administration. If HSR occurred during the administration of oxaliplatin or prior to administration of the next drug in the regimen's sequence, then oxaliplatin was identified as the causative agent. If HSR occurred after administration of subsequent drugs, then oxaliplatin

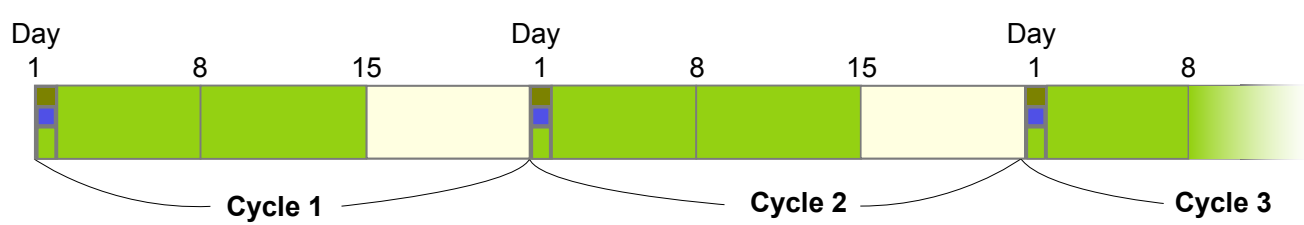

Premedication: palonosetron $0.75 \mathrm{mg}$ + dexamethasone $6.6 \mathrm{mg}$ + saline $20 \mathrm{~mL}$

BV $7.5 \mathrm{mg} / \mathrm{kg}+$ saline $100 \mathrm{~mL}$

30-90 minutes IV infusion

Oxaliplatin $130 \mathrm{mg} / \mathrm{m}^{2}+5 \%$ glucose $250 \mathrm{~mL}+$ dexamethasone $6.6 \mathrm{mg}$

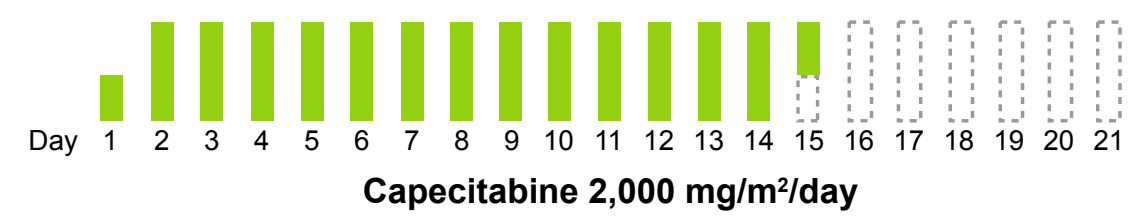

Figure I XELOX + bevacizumab regimen in this study.

Abbreviations: BV, bevacizumab; IV, intravenous; XELOX, capecitabine plus oxaliplatin. 
hypersensitivity was confirmed if there was a recurrence of symptoms, despite eliminating other suspected drugs.

\section{Results}

\section{Baseline patient characteristics}

Seventy-three patients were enrolled in this study between January and September 2013. Characteristics of the study patients are shown in Table 1 . Of the 73 patients, 31 received XELOX and 42 received XELOX plus bevacizumab. The median age was 64 years (range: $31-84$ ) and $52.1 \%$ were female. Eastern Cooperative Oncology Group performance status scores were 0,1 , and 2 in $83.6 \%, 13.7 \%$, and $2.7 \%$ of the patients, respectively. Twenty-eight patients received XELOX as part of adjuvant chemotherapy.

\section{Treatment}

The median number of treatment cycles was eight (range: 1-33). The cumulative median dose of oxaliplatin was $851 \mathrm{mg} / \mathrm{m}^{2}$.

Fifty-three patients $(72.6 \%)$ continued treatment until eight cycles, while the other reasons for discontinuing treatment were adverse events in four patients, operation in four patients, $\mathrm{CR}$ in one patient, progression disease in two patients, and refusal or personal reasons in nine patients.

Treatment was delayed in 22 patients $(30.1 \%)$ with the delay being due to neutropenia in four patients $(5.5 \%)$, thrombocytopenia in five, fatigue in three, diarrhea in two, PSN in one, pneumonia in one, cholangitis in one, ileus in one, and personal reasons in two patients. Twenty-three patients $(31.5 \%)$ required dose reduction at least once within eight cycles, with the reason being neutropenia in four and thrombocytopenia in five.

\section{Hypersensitivity reaction}

Three patients $(4.1 \%)$ developed HSR at five cycles, six cycles, and ten cycles. In all three cases, the reaction was cutaneous (grade 1 erythema) and it resolved within minutes

Table I Baseline characteristics of patients who received chemotherapy

\begin{tabular}{ll}
\hline XELOX + BV/XELOX & $42 / 31$ \\
Median age (range) & $64(31-84)$ \\
Male/female & $35 / 38$ \\
ECOG PS 0/I/2 & $61 / 10 / 2$ \\
Primary tumor colon/rectum & $37 / 36$ \\
Stage II/III/IV & $3 / 25 / 45$ \\
Oxaliplatin & $851 \pm 228.9 \mathrm{mg} / \mathrm{m}^{2}$ \\
\hline
\end{tabular}

Abbreviations: BV, bevacizumab; ECOG PS, Eastern Cooperative Oncology Group performance status; XELOX, capecitabine plus oxaliplatin. or hours. Two patients underwent oxaliplatin rechallenge. The reason for rechallenge treatment discontinuation of two patients was disease progression.

None of the 73 patients developed respiratory symptoms, ocular symptoms, or anaphylaxis.

\section{Efficacy}

The tumor response was assessed in 45 of the 73 patients because 28 patients received chemotherapy as part of adjuvant chemotherapy. The confirmed response rate was 64.4\% (95\% confidence interval [CI] 48.8-78.1; CR, 8.9\%; PR, 55.6\%; SD, 31.1\%; progressive disease [PD], 4.4\%), and disease control rate was achieved in $95.6 \%(95 \%$ CI 84.9-99.5).

\section{Safety}

Adverse events for 73 patients are summarized in Table 2. Two patients refused to continue the protocol treatment after the initial cycle. Grade 3 or higher hemotoxicity occurred in ten patients (13.7\%), and ten patients (13.7\%) developed grade 3 or higher nonhematological toxicity. There were no other severe treatment-related adverse events and no deaths during treatment.

\section{Discussion}

The incidence of HSRs to oxaliplatin is varying from $10 \%$ to $23.8 \% .^{3,5,14-17,23-28}$ Identifying patients at increased risk of oxaliplatin-induced HSRs is important and several studies have assessed risk factors, although with variable results. ${ }^{14,15,17,24,28}$ The mechanisms responsible for hypersensitivity to oxaliplatin remain unclear. ${ }^{29-32}$ However, it is likely that reactions to oxaliplatin are a type I, IgE-mediated hypersensitivity, while hemolysis and thrombocytopenia represent type II reactions and chronic urticaria, joint pain, and proteinuria are type III reactions. ${ }^{14,15,17,24,28}$ A review of the literature found that HSRs usually develop after multiple infusions of oxaliplatin, which clearly show that repeated

Table 2 Hematological and nonhematological adverse events

\begin{tabular}{ll}
\hline Hematological G3-4 & Nonhematological G3-4 \\
\hline I3.7\% & $13.7 \%$ \\
Neutropenia: 4 & Fatigue: 3 \\
Thrombocytopenia: 5 & Diarrhea: 2 \\
Aspartate aminotransferase: 3 & Nausea: 2 \\
& Vomiting: I \\
& Pneumonia: I \\
& Cholangitis: I \\
& lleus: I \\
& Neuropathy: I \\
\hline
\end{tabular}


exposure to the drug is a prerequisite to the induction of an allergic immune response. ${ }^{33}$ In addition to the review, there have been several case reports describing HSRs to oxaliplatin. $^{34-36}$

Kim et $\mathrm{a}^{17}$ reported that the antivascular epithelial growth factor monoclonal antibody bevacizumab was not associated with HSRs when administered with combination chemotherapy regimens. Consistent with their results, we found no difference in the frequency of HSRs according to the presence or absence of bevacizumab. There have been various approaches used to avoid HSRs to platinum-based agents, including premedication with high-dose corticosteroid ${ }^{37-39}$ desensitization procedures, ${ }^{40,41}$ and a slower infusion rate. ${ }^{40,42}$ At present, there is no standardized method for premedication to reduce the risk of oxaliplatin hypersensitivity. One study reported that premedication with a high-dose steroid (DEX $20 \mathrm{mg}$ ) significantly lowered the occurrence of oxaliplatin hypersensitivity and the rate of withdrawal from chemotherapy compared with premedication with the standard dose of steroid (DEX $8 \mathrm{mg}$ ). ${ }^{37}$ Therefore, an appropriate premedication steroid dose should be established for effective management of oxaliplatin hypersensitivity.

As shown in Table 3, the findings of the AVOID trial indicate that HSRs to oxaliplatin were low compared with other studies. We excluded patients with drug allergy and this could be an important bias. The $95 \% \mathrm{CI}$ is $0.9 \%-11.5 \%$ and the expected HSRs and threshold HSRs were $4 \%$ and $12 \%$, respectively. This has proven significantly that the HSRs incidence is less than $12 \%$. This may be simply because of the higher dosage of DEX, not because of the mixture; however, a recent study reported that a lower dose of DEX $(<20 \mathrm{mg})$ could be a positive risk factor. ${ }^{16}$ The change in $\mathrm{pH}$ caused by coinfusion of DEX may be one of the reasons. Histamine release was greatly influenced by $\mathrm{pH} ;{ }^{43-46}$ however, in investigating this effect it is necessary to avoid extreme changes in $\mathrm{pH}$ because such changes also cause a direct release of histamine. The curve for histamine release in response to an antigen has a minimum at $\mathrm{pH}$ 6.2-6.5. The kinetics of the alkaline hydrolysis of oxaliplatin has been evaluated. At $\mathrm{pH} 7.4$, a small fraction (maximally $0.7 \%$ ) of oxaliplatin will exist as the monodentate intermediate. However, it could rapidly react with endogenous compounds, resulting in a continuous conversion of oxaliplatin via the monodentate form. ${ }^{47} \mathrm{We}$ have previously reported changes in $\mathrm{pH}$ at $0,1,2$, and 3 hours after adding DEX (3.3 or $6.6 \mathrm{mg}$ ) to $250 \mathrm{~mL}$ of infusion solution containing $135 \mathrm{mg} / \mathrm{m}^{2}$ oxaliplatin. The $\mathrm{pH}$ without $\mathrm{DEX}$ was 4.78 , but increased to 6.90 and to 7.33 when DEX 3.3 and 6.6 mg, respectively, was added to oxaliplatin. ${ }^{12}$ The $\mathrm{pH}$ did not exceed 7.4 even when DEX was added to oxaliplatin. Most of oxaliplatin does not appear to be degraded when administered in combination with DEX. ${ }^{47}$ Therefore, the $\mathrm{pH}$ change caused by the coinfusion of DEX may reduce oxaliplatin-related HSRs. The XELOX regimen with DEX appears to be beneficial in the treatment of colorectal cancer, reducing the incidence of HSRs while maintaining an acceptable response rate.

We performed a premedication with steroids and palonosetron before the coinfusion of DEX and oxaliplatin. This is to prevent nausea and vomiting not to prevent HSR to oxaliplatin. A randomized trial that compare patients with and without premedication is mandatory. This trial is a single arm, but is the important trial that can lead to the next Phase III trial.

\section{Conclusion}

This findings of the AVOID trial suggest that the coinfusion of DEX and oxaliplatin can reduce oxaliplatin-induced HSRs. This approach should be considered for all patients who receive oxaliplatin, allowing treatment to be completed

Table 3 Comparison with other studies

\begin{tabular}{|c|c|c|c|c|}
\hline Study & $\begin{array}{l}\text { No of } \\
\text { patients }\end{array}$ & $\begin{array}{l}\text { Oxaliplatin } \\
\left(\mathrm{mg} / \mathrm{m}^{2}\right)\end{array}$ & $\begin{array}{l}\text { Hypersensitivity } \\
\text { all grade (\%) } \\
(95 \% \mathrm{Cl})\end{array}$ & $\begin{array}{l}\text { Hypersensitivity } \\
\text { G3-4 (\%) }\end{array}$ \\
\hline Brandi et $\mathrm{a}^{26}$ & 124 & - & | 3.7 (8.2-2|.0) & 7.3 \\
\hline Andre et $\mathrm{al}^{3}$ & $\mathrm{I}, 108$ & 820 & $10.3(8.6-12.2)$ & 2.9 \\
\hline Gowda et $\mathrm{al}^{27}$ & 169 & - & $18.9(13.3-25.7)$ & $<1$ \\
\hline Siu et $\mathrm{al}^{24}$ & 180 & - & $15(|0|-.2||)$. & 2.2 \\
\hline \multirow[t]{2}{*}{ Shibata et $\mathrm{al}^{28}$} & 125 & FOLFOX & $16.8(10.7-24.5)$ & 4 \\
\hline & & 8 cycles & & \\
\hline Seki et $\mathrm{al}^{25}$ & 108 & 560 & $22.3(|4.8-3| .2)$ & 9.3 \\
\hline Parel et $\mathrm{al}^{5}$ & 191 & 546 & $8.9(5.3-13.9)$ & 1.6 \\
\hline Our study & 73 & 851 & $4.1(0.9-11.5)$ & 0 \\
\hline
\end{tabular}

Abbreviations: $\mathrm{Cl}$, confidence interval; FOLFOX, folinic acid, fluorouracil, and oxaliplatin. 
as planned, In addition, we recommend our modified premedication regimen to reduce HSRs in clinical practice. However, Phase III prospective studies are warranted to confirm the safety and efficacy of this method.

\section{Acknowledgments}

We thank the participating patients, their family members, and all researchers involved in this study. We are grateful to Drs Tetsuo Shinohara and Takafumi Maekawa for their support as the independent review committee, and to Aya Tanaka for collecting data. This study was presented as a poster at the 2015 Gastrointestinal Cancers Symposium (http://meetinglibrary.asco.org/content/139904-158), and this paper is the first to publish the overall results as an original full-length article.

\section{Disclosure}

The authors report no conflicts of interest in this work.

\section{References}

1. de Gramont A, Figer A, Seymour M, et al. Leucovorin and fluorouracil with or without oxaliplatin as first-line treatment in advanced colorectal cancer. J Clin Oncol. 2000;18(16):2938-2947.

2. Tournigand $\mathrm{C}$, Andre T, Achille E, et al. FOLFIRI followed by FOLFOX6 or the reverse sequence in advanced colorectal cancer: a randomized GERCOR study. J Clin Oncol. 2004;22(2):229-237.

3. Andre T, Boni C, Mounedji-Boudiaf L, et al. Oxaliplatin, fluorouracil, and leucovorin as adjuvant treatment for colon cancer. $N$ Engl J Med. 2004;350(23):2343-2351.

4. Petrioli R, Pascucci A, Francini E, et al. Neurotoxicity of FOLFOX-4 as adjuvant treatment for patients with colon and gastric cancer: a randomized study of two different schedules of oxaliplatin. Cancer Chemother Pharmacol. 2008;61(1):105-111.

5. Parel M, Ranchon F, Nosbaum A, et al. Hypersensitivity to oxaliplatin: clinical features and risk factors. BMC Pharmacol Toxicol. 2014;15:1.

6. Kiernan MC, Krishnan AV. The pathophysiology of oxaliplatin-induced neurotoxicity. Curr Med Chem. 2006;13(24):2901-2907.

7. Jordan K, Behlendorf T, Surov A, Kegel T, Maher G, Wolf HH. Venous access ports: frequency and management of complications in oncology patients. Onkologie. 2008;31(7):404-410.

8. Kuwahara T, Asanami S, Kubo S. Experimental infusion phlebitis: tolerance osmolality of peripheral venous endothelial cells. Nutrition. 1998;14(6):496-501.

9. Nakayama S, Matsubara N, Sakai T, Aso N. [The incidence of phlebitis in the patients administrated vinorelbine by intravenous bolus injection-a retrospective study]. Gan To Kagaku Ryoho. 2002;29(4):633-635.

10. Curran CF, Luce JK, Page JA. Doxorubicin-associated flare reactions. Oncol Nurs Forum. 1990;17(3):387-389.

11. Matsuyama K, Mishima H, Ueno H, et al. [Etiology and management of venous pain during intravenous administration of oxaliplatin]. Gan To Kagaku Ryoho. 2011;38(3):411-414.

12. Yoshida Y, Hoshino S, Aisu N, et al. Dexamethasone as a means not only for controlling vascular pain caused by the administration of oxaliplatin via the peripheral vein but also for controlling oxaliplatin-induced hypersensitivity reactions. Br J Med Med Res. 2012;2(2):132-141.

13. Okayama T, Ishikawa T, Sugatani K, et al. Hypersensitivity reactions to oxaliplatin: identifying the risk factors and judging the efficacy of a desensitization protocol. Clin Ther. 2015;37(6):1259-1269.
14. Shao YY, Hu FC, Liang JT, Chiu WT, Cheng AL, Yang CH. Characteristics and risk factors of oxaliplatin-related hypersensitivity reactions. J Formos Med Assoc. 2010;109(5):362-368.

15. Mori Y, Nishimura T, Kitano T, et al. Oxaliplatin-free interval as a risk factor for hypersensitivity reaction among colorectal cancer patients treated with FOLFOX. Oncology. 2010;79(1-2):136-143.

16. Kim MY, Kang SY, Lee SY, et al. Hypersensitivity reactions to oxaliplatin: clinical features and risk factors in Koreans. Asian Pac J Cancer Prev. 2012;13(4):1209-1215.

17. Kim BH, Bradley T, Tai J, Budman DR. Hypersensitivity to oxaliplatin: an investigation of incidence and risk factors, and literature review. Oncology. 2009;76(4):231-238.

18. Yoshida Y, Hoshino S, Aisu N, et al. Pilot study of the early start of chemotherapy after resection of primary colorectal cancer with distant metastases (Pearl Star 01). World J Surg Oncol. 2013;11(1):39.

19. Yoshida $Y$, Hoshino S, Aisu N, et al. Administration of chemotherapy via the median cubital vein without implantable central venous access ports: port-free chemotherapy for metastatic colorectal cancer patients. Int J Clin Oncol. 2015;20(2):332-337.

20. Yoshida Y, Hoshino S, Aisu N, et al. Can grade 2 neutropenia predict the risk of grade 3 neutropenia in metastatic colorectal cancer patients treated with chemotherapy? Support Care Cancer. 2015;23(6):1623-1627.

21. Demoly P, Adkinson NF, Brockow K, et al. International Consensus on drug allergy. Allergy. 2014;69(4):420-437.

22. Johansson SG, Bieber T, Dahl R, et al. Revised nomenclature for allergy for global use: Report of the Nomenclature Review Committee of the World Allergy Organization, October 2003. J Allergy Clin Immunol. 2004;113(5):832-836.

23. Ichikawa Y, Goto A, Hirokawa S, et al. Allergic reactions to oxaliplatin in a single institute in Japan. Jpn J Clin Oncol. 2009;39(9):616-620.

24. Siu SW, Chan RT, Au GK. Hypersensitivity reactions to oxaliplatin: experience in a single institute. Ann Oncol. 2006;17(2):259-261.

25. Seki K, Senzaki K, Tsuduki Y, et al. Risk factors for oxaliplatin-induced hypersensitivity reactions in Japanese patients with advanced colorectal cancer. Int J Med Sci. 2011;8(3):210-215.

26. Brandi G, Pantaleo M, Galli C, et al. Hypersensitivity reactions related to oxaliplatin (OHP). Br J Cancer. 2003;89(3):477-481.

27. Gowda A, Goel R, Berdzik J, Leichman CG, Javle M. Hypersensitivity reactions to oxaliplatin: incidence and management. Oncology (Williston Park, NY). 2004;18(13):1671-1675; discussion 1676, 1680, 1683-1674.

28. Shibata Y, Ariyama H, Baba E, et al. Oxaliplatin-induced allergic reaction in patients with colorectal cancer in Japan. Int J Clin Oncol. 2009;14(5):397-401.

29. Yanai T, Iwasa S, Hashimoto H, et al. Successful rechallenge for oxaliplatin hypersensitivity reactions in patients with metastatic colorectal cancer. Anticancer Res. 2012;32(12):5521-5526.

30. Castells M, Sancho-Serra Mdel C, Simarro M. Hypersensitivity to antineoplastic agents: mechanisms and treatment with rapid desensitization. Cancer Immunol Immunother. 2012;61(9):1575-1584.

31. Cortijo-Cascajares S, Nacle-Lopez I, Garcia-Escobar I, et al. Effectiveness of oxaliplatin desensitization protocols. Clin Transl Oncol. 2013;15(3):219-225.

32. Couraud S, Planus C, Rioufol C, Mornex F. [Platinum salts hypersensitivity]. Rev Pneumol Clin. 2008;64(1):20-26.

33. Maindrault-Goebel F, Andre T, Tournigand C, et al. Allergic-type reactions to oxaliplatin: retrospective analysis of 42 patients. Eur $J$ Cancer. 2005;41(15):2262-2267.

34. Thomas RR, Quinn MG, Schuler B, Grem JL. Hypersensitivity and idiosyncratic reactions to oxaliplatin. Cancer. 2003;97(9):2301-2307.

35. Syrigou E, Syrigos K, Saif MW. Hypersensitivity reactions to oxaliplatin and other antineoplastic agents. Curr Allergy Asthma Rep. 2008;8(1):56-62.

36. Saadati H, Saif MW. Oxaliplatin-induced hyperexcitability syndrome in a patient with pancreatic cancer. JOP. 2009;10(4): 459-461. 
37. Kidera Y, Satoh T, Ueda S, et al. High-dose dexamethasone plus antihistamine prevents colorectal cancer patients treated with modified FOLFOX6 from hypersensitivity reactions induced by oxaliplatin. Int J Clin Oncol. 2011;16(3):244-249.

38. Thomas RR, Quinn MG, Schuler B, Grem JL. Hypersensitivity and idiosyncratic reactions to oxaliplatin. Cancer. 2003;97(9):2301-2307.

39. Suenaga M, Mizunuma N, Shinozaki E, et al. Management of allergic reactions to oxaliplatin in colorectal cancer patients. J Support Oncol. 2008;6(8):373-378.

40. Mis L, Fernando NH, Hurwitz HI, Morse MA. Successful desensitization to oxaliplatin. Ann Pharmacother. 2005;39(5):966-969.

41. Goldberg A, Confino-Cohen R, Fishman A, Beyth Y, Altaras M. A modified, prolonged desensitization protocol in carboplatin allergy. J Allergy Clin Immunol. 1996;98(4):841-843.

42. Bhargava P, Gammon D, McCormick MJ. Hypersensitivity and idiosyncratic reactions to oxaliplatin. Cancer. 2004;100(1):211-212.
43. Mongar J, Schild $\mathrm{H}$. The effect of calcium and $\mathrm{pH}$ on the anaphylactic reaction. J Physiol. 1958;140(2):272-284.

44. Jensen TB, Friis UG, Johansen T. Role of physiological HCO3-buffer on intracellular $\mathrm{pH}$ and histamine release in rat peritoneal mast cells. Pflugers Arch. 1998;436(3):357-364.

45. Bitziou E, O'Hare D, Patel BA. Simultaneous detection of $\mathrm{pH}$ changes and histamine release from oxyntic glands in isolated stomach. Anal Chem. 2008;80(22):8733-8740.

46. Lober K, Alfonso A, Escribano L, Botana LM. STI571 (Glivec) affects histamine release and intracellular $\mathrm{pH}$ after alkalinisation in HMC-1560, 816. J Cell Biochem. 2008;103(3):865-876.

47. Jerremalm E, Eksborg S, Ehrsson H. Hydrolysis of oxaliplatin-evaluation of the acid dissociation constant for the oxalato monodentate complex. J Pharm Sci. 2003;92(2):436-438.

\section{Publish your work in this journal}

Drug Design, Development and Therapy is an international, peerreviewed open-access journal that spans the spectrum of drug design and development through to clinical applications. Clinical outcomes, patient safety, and programs for the development and effective, safe, and sustained use of medicines are a feature of the journal, which has also been accepted for indexing on PubMed Central. The manuscript management system is completely online and includes a very quick and fair peer-review system, which is all easy to use. Visit http://www.dovepress.com/testimonials.php to read real quotes from published authors.

Submit your manuscript here: http://www.dovepress.com/drug-design-development-and-therapy-journal 